\title{
Unraveling the role of the gut microbiota in obesity; cause or effect?
}

\author{
M. J. Khan ${ }^{1}$, V. Svolos ${ }^{1}$, S. Cheng ${ }^{1}$, G. M. Shaikh ${ }^{2}$, C. Quince ${ }^{3}$, C. A. Edwards ${ }^{1}$ \\ and K. Gerasimidis ${ }^{1}$ \\ ${ }^{1}$ Human Nutrition College of Medicine, Veterinary and Life Sciences, University of Glasgow, G3 8SJ, \\ ${ }^{2}$ Department of Endocrinology, Royal Hospital for Sick Children Yorkhill, Glasgow G3 8SJ and \\ ${ }^{3}$ School of Engineering, University of Glasgow, G12 $8 Q Q$
}

The gut microbiota has been implicated in the aetiology of obesity ${ }^{(1)}$. However the evidence is inconclusive as to whether differences between lean and obese people are a cause of obesity or if it is an effect of different dietary patterns between lean and obese individuals $^{(2)}$. We explored the possibility of reverse causality by comparing gut microbial composition and bacterial metabolic activity in children with obesity of different aetiology.

Faecal samples were collected from children/young adults with 'simple' obesity $(n=17)$, hypothalamic obese ( $n=12$ with Prader-Willi syndrome), hypothalamic lean $(n=10$ with Prader-Willi) and healthy lean controls $(n=20)$. Faecal samples were subjected to deep sequencing of the 16S rDNA with the 454 platform. Faecal short chain fatty acids (SCFA), sulphide and ammonia were measured. The fermentative capacity of the gut microbiota from each subject group was assessed with $24 \mathrm{~h}$ in-vitro batch culture fermentations using 5 different fermentable carbohydrates (Apple pectin, raw potato starch, wheat bran, raftilose and maize starch).

There was no difference in faecal SCFA concentration between children with obesity of different aetiology. Obese ('simple' \& hypothalamic together) children had significantly higher concentration of propionate than lean (control \& lean hypothalamic together) children $(72.7 \mu \mathrm{mol} / \mathrm{g}$ freeze dried faecal material $v s .51 .1 \mu \mathrm{mol} / \mathrm{g}, p=0.008)$. Total SCFA concentration was positively correlated with BMI $z$-score (Spearman correlation $=0.21, p=0.03$ ). Faecal $\mathrm{pH}$, ammonia, and free and total sulphide were not significantly different between the different phenotypes and aetiology of obesity. No major differences were observed in the $24 \mathrm{~h}$ fermentation capacity of the microbiota of the four groups. There was no significant difference in the relative abundance of the gut microbiota between children with obesity of different aetiology. Relative abundance of Bacteroidetes (13\% vs. 6.8\%) and Ruminococcus ( $5 \%$ vs. $1.8 \%$ ) were significantly higher $(p<0.05)$, while Anaerostipes $(0.9 \%$ vs. 3.4\%), Eubacterium $(0.7 \%$ vs. $2.2 \%)$, Lactobacillus (0.2\% vs. $0.9 \%)$, Lactococcus $(0.1 \%$ vs. $0.6 \%)$, and Asaccharbacter $(0.1 \%$ vs. $0.6 \%)$ were significantly lower $(p<0.05)$ in the obese vs. lean group.

These results do not support the role of the gut microbiota in the aetiology of obesity but provide strong evidence to suggest that the findings reported in previous studies are likely to be the result of hyperphagia and different dietary patterns between lean and obese children.

This study is part of a PhD project funded by Khyber Medical University Peshawar, Pakistan and Yorkhill Children Charity, Royal Hospital for Sick Children Yorkhill, Glasgow G3 8SJ UK.

1. Tremaroli V, Kovatcheva-Datchary P, Backhed F (2010) Gut 59, 1589-90.

2. Greiner T, Backhed F (2011) Trends Endocrinol Metab 22, 117-23. 